\title{
REVIEWS
}

Adv Clin Exp Med 2015, 24, 1, 161-165

DOI: $10.17219 /$ acem/38144

(C) Copyright by Wroclaw Medical University

ISSN 1899-5276

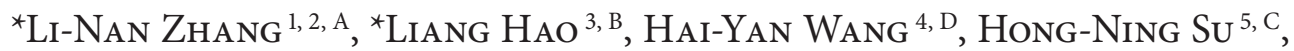

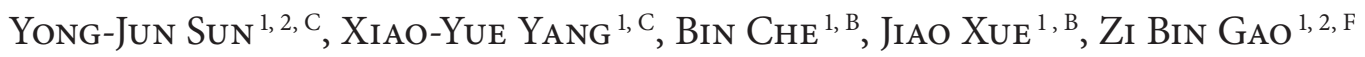

\section{Neuroprotective Effect of Resveratrol Against Glutamate-Induced Excitotoxicity}

${ }^{1}$ Pharmacy Department, Hebei University of Science and Technology, Shijiazhuang, Hebei, China

2 State Key Laboratory Breeding Base, Hebei Province Key Laboratory of Molecular Chemistry for Drugs, Shijiazhuang, Hebei, China

${ }^{3}$ Department of Neurosurgery, Third Hospital of Shijiazhuang, Shijiazhuang, Hebei, China

${ }^{4}$ Pharmaceutical Preparation Section, Third Hospital of Shijiazhuang, Shijiazhuang, Hebei, China

${ }^{5}$ Pharmaceutical Preparation Section, Fourth Hospital of Shijiazhuang, Shijiazhuang, Hebei, China

A - research concept and design; B - collection and/or assembly of data; C - data analysis and interpretation;

$\mathbf{D}$ - writing the article; $\mathbf{E}$ - critical revision of the article; $\mathbf{F}$ - final approval of article; $\mathbf{G}$ - other

\begin{abstract}
As the major neurotransmitter in the mammalian central nervous system (CNS), excessive extracellular glutamate (Glu) can activate the Glu receptors and neuronal calcium $(\mathrm{Ca} 2+)$ overload, then produce neurotoxicity, which is a common pathway for neuronal injury or death, and is associated with acute and chronic neurodegenerative diseases. Therefore, it has been a therapeutic strategy to investigate neuroprotective effects against Glu-induced neurotoxicity for treating both acute and chronic forms of neurodegeneration. Resveratrol (Res), as a naturally occurring polyphenol mainly found in grapes and red wine, has shown a neuroprotective effect in a variety of experimental models for neurodegenerative diseases in vitro and in vivo. This review will focus on the neuroprotective effect of Res against Glu-induced excitotoxicity in neurodegenerative diseases by blocking different Glu receptors and Ca2+ ion channels (Adv Clin Exp Med 2015, 24, 1, 161-165).
\end{abstract}

Key words: resveratrol, glutamate, excitotoxicity, neuroprotective effect.

Glutamate (Glu) is the major excitatory neurotransmitter in the central nervous system (CNS), involved fast synaptic transmission, neuronal plasticity, outgrowth and survival, memory, learning and behavior [1]. Apart from its physiological role, excessive Glu leads to activation of the Glu receptors, particularly of $\mathrm{N}$-methyl-D-aspartic acid (NMDA), a-amino-3-hydroxy-5-methylisoxazole-4-propionate (AMPA) and kainic acid (KA) subtypes; to excessive Ca2+ influx into neurons through ionic channels, and secondary excitotoxicity [2]; and finally to neuronal dysfunction, damage or even death [3]. As the final common pathway for neurologic disorders, Glumediated neurotoxicity has been shown to contribute to pathological consequences in several neuropathological disorders, particularly in Alzheimer's disease, Parkinson's disease, epilepsy, ischemic stroke and major depression [4]. For this reason, it is important to understand the various types of Glu receptors and the ion channels that they are capable of activating either directly or indirectly. Early work on Glu-induced excitotoxicity emphasized the importance of KA and its receptor in producing this type of neuronal damage [5]. Other studies have shown that a specific blockade of the NMDA receptor largely blocks the toxicity of exogenous Glu in cultured cortical neurons $[3,6]$. The most relevant biochemical events in Glu-mediated neurotoxicity might be the acute influx of extracellular $\mathrm{Ca} 2+$ through cell membrane channels. However, other routes, including L- and

* Li-Nan Zhang and Liang Hao contributed equally to this work. 
$\mathrm{N}$-type voltage-gated $\mathrm{Ca} 2+$ channels, the $\mathrm{Na}+$ / $/ \mathrm{Ca} 2+$ exchanger, and non-specific membrane leak, may also participate [7].

Resveratrol (Res) has been shown to exhibit various beneficial biological effects, such as protective effects against cardiovascular diseases [8], anti-inflammatory or anticarcinogenic activities [9]. Res has also been shown to pass the blood brain barrier and exert neuroprotective effect, and therefore its use for the prevention and treatment of neurodegenerative diseases, such as cerebral ischemia, Alzheimer's disease, Parkinson's disease and amyotrophic lateral sclerosis, has been investigated [10]. For example, Res protected against neurotoxic effects and antagonized the $[\mathrm{Ca} 2+]$ i elevation produced by excess Glu concentrations in cerebrospinal fluid from amyotrophic lateral sclerosis patients, primary cultured rat cortical cells and the murine hippocampal cell line HT 22 [11-13]. Recent studies have reported that Res increased Glu uptake, glutathione content and S 100B secretion in primary cortical astrocytes [14], and reduced Glu-induced radical formation in murine brain cultures [15]. Res and capsaicin combined enhance neuroprotection against Glu-induced toxicity in mouse cerebral cortical neurons, implying a useful therapeutic method for treating neurodegenerative disorders [16]. A recent study confirmed that trans-Res significantly suppressed Glu-induced currents in rat hippocampal postsynaptic CA1 pyramidal neurons (which may be due to the L-type $\mathrm{Ca} 2+$ channel blockade and a subsequent reduction of $\mathrm{Ca} 2+$ influx) and had antioxidant action that ameliorated ischemic brain injury [17]. These findings support the use of Res in the treatment of diseases induced by Glu-mediated exitotoxicity in the future.

\section{Res-Mediated Neuroprotective Effects Against Glu-Induced Excitotoxicity}

Res inhibits the increase of extracellular Glu levels induced by stretch injuries by enhancing the Glu transporter, thus increasing Glu uptake, glutathione content, glutamine synthetase activity and S 100B (a neurotrophic cytokine) secretion in cortical astrocyte cultures and C6 glioma cells $[14,18,19]$. Moreover, Res could directly inhibit Glu release in rat cerebrocortical nerve terminals by decreasing mitogen-activated protein kinase activation and, subsequently suppressing voltage-dependent $\mathrm{Ca} 2+$ channel activity [20]. The key reason for Glu-induced excitotoxicity is that Glu can markedly activate Glu receptors, and increase the concentration of intracellular $\mathrm{Ca} 2+([\mathrm{Ca} 2+] \mathrm{i})$ in neurons, and thus finally induce neuronal death. The major ionotropic receptors activated by Glu are commonly referred to as NMDA, AMPA and KA receptors.

\section{Res-Mediated Neuroprotective Effects Against Glu-Induced Excitotoxicity via NMDA Receptors}

Glu-induced excitotoxicity is closely related to the activation of NMDA receptors [21, 22]. The NMDA-mediated entry of excessive $\mathrm{Ca} 2+$ into the cytosol activates calcineurin, which induces apoptosis in both rat hippocampal neurons and glioblastoma cells $[23,24]$ via voltage-dependent $\mathrm{Ca} 2+$ channels and NMDA receptor-operated channels [25]. Therefore, current efforts to develop an effective therapy for hypoxic-ischemic neuronal injury are appropriately focused on NMDA antagonists and $\mathrm{Ca} 2+$ blockers [7]. The uncompetitive NMDA receptor antagonist memantine, is able to protect dissociated cortical neurons from Glu-induced excitotoxicity [26]. Res attenuates oxygen-glucose deprivationinduced neuron impairment and death in acute rat hippocampal slices by enhancing the activation of the large-conductance potassium channel and reducing the enhanced AMPA/NMDA receptor-mediated neuronal excitatory postsynaptic currents caused by oxygen-glucose deprivation [27]. Furthermore, some studies support that trans-Res inhibites the postsynaptic Glu receptors in hippocampal neurons, with NMDA receptors being more sensitive than AMPA receptors [17], which probably have an antioxidant effect that ameliorates ischemic brain injury. Res also inhibited NMDA-induced neuronal death, intracellular calcium increase and reactive oxygen species generation in rat cortical neurons, confirming the neuroprotective effect of Res $[11,28]$. However, other studies have shown that pretreatment with Res did not alter the magnitude of the hypothalamus-pituitary-adrenal axis response to NMDA [29]. Furthermore, some studies have shown that treating neurons with Res did not protect them from Glu/NMDA-induced depletion of nicotinamide adenine dinucleotide (NAD+), an important energy substrate and cofactor in multiple metabolic reactions, and death [30]. These findings suggest that Res protection 
against Glu-induced excitotoxicity via NMDA receptors is still controversial, and further studies should be undertaken.

\section{Res-Mediated Neuroprotective Effect Against Glu-Induced Excitotoxicity via AMPA Receptors}

Res has been shown to reduce p 38 mitogenactivated protein kinase (MAPK) activation in cultured astrocytes following a stretch injury; the AMPA receptor is involved in p 38 MAPK activation following an injury [18]. Res also markedly reduced the amplitude and frequency of AMPA-mediated spontaneous excitatory postsynaptic currents in rat hippocampal pyramidal neurons [27]. Furthermore, trans-Res significantly suppressed Glu-induced currents in rat hippocampal postsynaptic CA1 pyramidal neurons, and NMDA and KA receptors were more sensitive than AMPA receptors to trans-Res [17].

\section{Res-Mediated Neuroprotective Effect Against Glu-Induced Excitotoxicity via KA Receptors}

Seizures induced by KA produced a marked increase in the free radical nitric oxide, causing oxidative stress and leading to depletion of energy stores [31]. Res or trans-Res has a neuroprotective effect against KA-induced convulsions and the attenuation of raised malondialdehyde levels, and markedly decreases spontaneous seizure frequency, suggesting that Res may be a potential anti-epilepsy agent, at least as an adjunct treatment in KA-induced epilepsy animal models $[32,33]$. The chronic administration of Res significantly protects against KA-induced damage in the rat hippocampus and olfactory cortex, whereas the same treatment with Res does not play a protective role in an ex vivo hippocampal ischemia model [34]. Furthermore, KA-induced excitotoxicity has been implicated in increased oxidative stress. For example, KA-induced hippocampal neuronal damage and activation of astrocytes and microglial cells were significantly attenuated by treatment with
Res, demonstrating that Res could act as free radical scavenger to protect against cell damage caused by excitotoxic insults $[35,36]$. Furthermore, KA receptors have been reported to be involved in trans-Res-induced inhibition of Glu-induced currents in rat postsynaptic CA1 pyramidal neurons [17]. These studies suggest that Res may play an important role in protection from KA receptorsinduced excitotoxicity.

\section{Discussion}

Generally, NMDA, AMPA and KA receptors can all be involved in Glu-induced excitotoxicity, separately or together, with varying sensitivity. For example, trans-Res significantly suppressed Glu-induced currents in rat postsynaptic CA1 pyramidal neurons, where KA and NMDA receptors were more sensitive to trans-Res than AMPA receptors [17]. Furthermore, Res induces opposite effects not only in different positions, but also at low vs high doses, indicative of the hormetic dose response [37]. For example, at a low concentration $(25 \mathrm{mM})$, Res protected neurons from being killed by Glu and NMDA, whereas high concentrations of Res either had no effect or exacerbated excitotoxic neuronal death [30]. Some studies have shown that capsaicin or Res significantly increases cell viability, and the co-treatment with capsaicin and Res has a more significant impact than capsaicin or Res alone. Furthermore, the reactive oxygen species generation and the sequent apoptotic neuronal death induced by Glu are also significantly decreased by the co-treatment with both capsaicin and Res, suggesting that co-treatment with capsaicin and Res enhances neuroprotection against Glu-induced toxicity in the mouse cortex [16]. These data suggest that Glu-induced excitotoxicity via Glu receptors are still controversial, needing further exploration.

Glu-induced excitotoxicity has been clearly attributed to a massive influx of $\mathrm{Ca} 2+$ through NMDA and non-NMDA channels [38]. NMDA receptor-mediated elevations in $[\mathrm{Ca} 2+] \mathrm{i}$ have been shown to induce "epilepsy" in cultured hippocampal neurons [39]. Res can significantly reduce epileptiform discharges induced by Glu and reverse the increased discharges induced by Bay K 8644, the selective L-type Ca2+ channel agonist, which strongly suggests that the inhibitory effects of Res might be due to a blockade of Ca2+ influx [40]. Other studies have reported that Res protects against Glu-independent neurotoxic effects and antagonizes the $[\mathrm{Ca} 2+]$ i elevation produced by cerebrospinal fluid from rat brain cortical motoneurons suffering amyotrophic lateral sclerosis [11]. 
Furthermore, Res does not protect against Glu/ /NMDA-induced NAD+ depletion and death in cultured neurons [30]. These studies may indicate the potential for Res to be used as a pharma- cological tool for the treatment of diseases induced by the Glu excitotoxicity. However, there are still some controversies that, needfurther exploration in the future.

Acknowledgements. The authors acknowledge the support received for this study from the Natural Science Foundation of China (NSFC 81402886), the Natural Science Foundation of Hebei Province (H 2014208004, H 2012208080), Hebei Education Department Science Foundation (QN 2014093), and the Hebei University of Science and Technology Discipline Construction Office and the State Key Laboratory Breeding Base-Hebei Key Laboratory of Molecular Chemistry for Drugs.

\section{References}

[1] Albright TD, Jessell TM, Kandel ER, Posner MI: Neural science: a century of progress and the mysteries that remain. Cell 2000, 100 Suppl, 1-55.

[2] Dong XX, Wang Y, Qin ZH: Molecular mechanisms of excitotoxicity and their relevance to pathogenesis of neurodegenerative diseases. Acta Pharmacol Sin 2009, 30, 379-387.

[3] Choi DW: Glutamate neurotoxicity and diseases of the nervous system. Neuron 1988, 1, 623-634.

[4] Lipton SA, Rosenberg PA: Excitatory amino acids as a final common pathway for neurologic disorders. N Engl J Med 1994, 330, 613-622.

[5] Coyle JT: Neurotoxic action of kainic acid. J Neurochem 1983, 41, 1-11.

[6] Finkbeiner S, Stevens CF: Applications of quantitative measurements for assessing glutamate neurotoxicity. Proc Natl Acad Sci USA 1988, 85, 4071-4074.

[7] Choi DW: Calcium-mediated neurotoxicity: relationship to specific channel types and role in ischemic damage. Trends Neurosci 1988, 11, 465-469.

[8] Sayin O, Arslan N, Guner G: The protective effects of resveratrol on human coronary artery endothelial cell damage induced by hydrogen peroxide in vitro. Acta Clin Croat 2012, 51, 227-235.

[9] Kim J, Lee HJ, Lee KW: Naturally occurring phytochemicals for the prevention of Alzheimer's disease. J Neurochem 2010, 112, 1415-1430.

[10] Robb EL, Stuart JA: trans-Resveratrol as a neuroprotectant. Molecules 2010, 15, 1196-1212.

[11] Yanez M, Galan L, Matias-Guiu J, Vela A, Guerrero A, Garcia AG: CSF from amyotrophic lateral sclerosis patients produces glutamate independent death of rat motor brain cortical neurons: protection by resveratrol but not riluzole. Brain Res 2011, 1423, 77-86.

[12] Chung IM, Yeo MA, Kim SJ, Moon HI: Neuroprotective effects of resveratrol derivatives from the roots of Vitis thunbergii var. sinuate against glutamate-induced neurotoxicity in primary cultured rat cortical cells. Hum Exp Toxicol 2011, 30, 1404-1408.

[13] Fukui M, Choi HJ, Zhu BT: Mechanism for the protective effect of resveratrol against oxidative stress-induced neuronal death. Free Radic Biol Med 2010, 49, 800-813.

[14] de Almeida LM, Pineiro CC, Leite MC: Resveratrol increases glutamate uptake, glutathione content, and S 100B secretion in cortical astrocyte cultures. Cell Mol Neurobiol 2007, 27, 661-668.

[15] Moldzio R, Radad K, Krewenka C, Kranner B, Duvigneau JC, Rausch WD: Protective effects of resveratrol on glutamate-induced damages in murine brain cultures. J Neural Transm 2013.

[16] Lee JG, Yon JM, Lin C, Jung AY, Jung KY, Nam SY: Combined treatment with capsaicin and resveratrol enhances neuroprotection against glutamate-induced toxicity in mouse cerebral cortical neurons. Food Chem Toxicol 2012, 50, 3877-3885.

[17] Gao ZB, Chen XQ, Hu GY: Inhibition of excitatory synaptic transmission by trans-resveratrol in rat hippocampus. Brain Res 2006, 1111, 41-47.

[18] Zhou H, Chen Q, Kong DL, Guo J, Wang Q, Yu SY: Effect of resveratrol on gliotransmitter levels and p 38 activities in cultured astrocytes. Neurochem Res 2011, 36, 17-26.

[19] dos Santos AQ, Nardin P, Funchal C: Resveratrol increases glutamate uptake and glutamine synthetase activity in C 6 glioma cells. Arch Biochem Biophys 2006, 453, 161-167.

[20] Chang Y, Wang SJ: Inhibitory effect of glutamate release from rat cerebrocortical nerve terminals by resveratrol. Neurochem Int 2009, 54, 135-141.

[21] Petroni D, Tsai J, Mondal D, George W: Attenuation of low dose methylmercury and glutamate induced-cytotoxicity and tau phosphorylation by an N-methyl-D-aspartate antagonist in human neuroblastoma (SHSY 5Y) cells. Environ Toxicol 2011.

[22] Tanovic A, Alfaro V: [Glutamate-related excitotoxicity neuroprotection with memantine, an uncompetitive antagonist of NMDA-glutamate receptor, in Alzheimer's disease and vascular dementia]. Rev Neurol 2006, 42, 607-616.

[23] Szado T, Vanderheyden V, Parys JB: Phosphorylation of inositol 1,4,5-trisphosphate receptors by protein kinase B/Akt inhibits Ca 2+ release and apoptosis. Proc Natl Acad Sci USA 2008, 105, 2427-2432.

[24] Wang HG, Pathan N, Ethell IM: Ca 2+-induced apoptosis through calcineurin dephosphorylation of BAD. Science 1999, 284, 339-343.

[25] Zeng Z, Li W, Yao Y, Chen N: Study on the mechanism of glutamate mediated neurotoxicity by cortical neuron culture technique in vitro. Hua Xi Yi Ke Da Xue Xue Bao 1999, 30, 329-330. 
[26] Kutzing MK, Luo V, Firestein BL: Protection from glutamate-induced excitotoxicity by memantine. Ann Biomed Eng 2011, 40, 1170-1181.

[27] Zhang H, Schools GP, Lei T, Wang W, Kimelberg HK, Zhou M: Resveratrol attenuates early pyramidal neuron excitability impairment and death in acute rat hippocampal slices caused by oxygen-glucose deprivation. Exp Neurol 2008, 212, 44-52.

[28] Ban JY, Cho SO, Choi SH: Neuroprotective effect of Smilacis chinae rhizome on NMDA-induced neurotoxicity in vitro and focal cerebral ischemia in vivo. J Pharmacol Sci 2008, 106, 68-77.

[29] Knutson N, Wood CE: Interaction of PGHS-2 and glutamatergic mechanisms controlling the ovine fetal hypothalamus-pituitary-adrenal axis. Am J Physiol Regul Integr Comp Physiol 2010, 299, 365-370.

[30] Liu D, Gharavi R, Pitta M, Gleichmann M, Mattson MP: Nicotinamide prevents NAD+ depletion and protects neurons against excitotoxicity and cerebral ischemia: NAD+ consumption by SIRT 1 may endanger energetically compromised neurons. Neuromolecular Med 2009, 11, 28-42.

[31] Milatovic D, Gupta RC, Dettbarn WD: Involvement of nitric oxide in kainic acid-induced excitotoxicity in rat brain. Brain Res 2002, 957, 330-337.

[32] Gupta YK, Briyal S, Chaudhary G: Protective effect of trans-resveratrol against kainic acid-induced seizures and oxidative stress in rats. Pharmacol Biochem Behav 2002, 71, 245-249.

[33] Wu Z, Xu Q, Zhang L, Kong D, Ma R, Wang L: Protective effect of resveratrol against kainate-induced temporal lobe epilepsy in rats. Neurochem Res 2009, 34, 1393-1400.

[34] Virgili M, Contestabile A: Partial neuroprotection of in vivo excitotoxic brain damage by chronic administration of the red wine antioxidant agent, trans-resveratrol in rats. Neurosci Lett 2000, 281, 123-126.

[35] Wang Q, Yu S, Simonyi A, Rottinghaus G, Sun GY, Sun AY: Resveratrol protects against neurotoxicity induced by kainic acid. Neurochem Res 2004, 29, 2105-2112.

[36] Friedman LK, Goldstein B, Rafiuddin A, Roblejo P, Friedman S: Lack of resveratrol neuroprotection in developing rats treated with kainic acid. Neuroscience 2013, 230, 39-49.

[37] Tedesco I, Russo M, Russo GL: Commentary on 'resveratrol commonly displays hormesis: occurrence and biomedical significance'. Hum Exp Toxicol 2010, 29, 1029-1031.

[38] Choi DW, Rothman SM: The role of glutamate neurotoxicity in hypoxic-ischemic neuronal death. Annu Rev Neurosci 1990, 13, 171-182.

[39] DeLorenzo RJ, Pal S, Sombati S: Prolonged activation of the N-methyl-D-aspartate receptor-Ca 2+ transduction pathway causes spontaneous recurrent epileptiform discharges in hippocampal neurons in culture. Proc Natl Acad Sci USA 1998, 95, 14482-14487.

[40] Li M, Wang QS, Chen Y, Wang ZM, Liu Z, Guo SM: Resveratrol inhibits the electrical activity of subfornical organ neurons in rat. Sheng Li Xue Bao 2005, 57, 523-528.

\section{Address for correspondence:}

Zibin Gao

Pharmacy Department

Hebei University of Science and Technology

70 Yuhua East Road

Shijiazhuang

Hebei 050018

China

Tel.: +8631188632427

E-mail: zbgao 74@163.com

Conflict of interest: None declared

Received: 5.06.2013

Revised: 17.01.2014

Accepted: 12.01.2015 